\title{
Amyloidosis in ENT: Review of the Literature and a Unique Rhinology Case
}

\author{
Salil Sood*, Eugene Omakobia, Miroslav Radojkovic and Deepak Gupta \\ ENT Fellow, Bradford Royal infirmary Bradford, UK
}

Submission: May 21, 2018; Published: June 05, 2018

*Corresponding author: Salil Sood, ENT Fellow, Bradford Royal infirmary Bradford, UK, Email: salilsood@hotmail.co.uk

Abstract

Amyloidosis is a diagnosis not commonly encountered by ENT clinicians. It is reported that $19 \%$ of cases involve the head and neck with the oropharynx and larynx being the most commonly affected areas. A high index of clinical suspicion is required to make the diagnosis. Here, we report an unusual case presenting in the nasopharynx. This case was particularly rare in that it clinically mimicked idiopathic midline destructive disease (IMDD). We have placed particular emphasis on the differential diagnoses which must be excluded and the radiological and histopathological features which must be elicited.

Abbreviations: WBC: White Blood Cell Count; ESR: Erythrocyte Sedimentation Rate; CRP: C-Reactive Protein; IMDD: Idiopathic Midline Destructive Disease; MRI: Magnetic Resonance Imaging

\section{Introduction}

Amyloidosis is a benign clinical condition characterised by abnormal deposition of a particular protein referred to as amyloid. It is comprised of linear, non-branching, fibrillar proteinaceous material arranged in highly organised fashion. On electron microscopy, amyloid deposits have a fibrous appearance with a cross linked, beta-pleated sheet conformation. Amyloidosis can be classified as primary (70\%), localised (19\%), familial (4\%) and secondary (3\%) [1]. 19\% of cases involve the head and neck with the oropharynx and larynx being the most affected organs. Systemic amyloidosis has 3 forms [2]:

a) AL amyloidosis- myeloma or plasma cell dyscrasia.

b) AA or reactive- a chronic inflammatory condition.

c) Familial amyloidosis- an autosomal recessive disorder.

Systemically, proteinuria is often the first symptom. Other manifestation may include peripheral neuropathy, dementia, cognitive dysfunction and organ dysfunction of the liver, kidney, heart or spleen. Of particular relevance to otolaryngologists, localised nasopharyngeal amyloidosis may present with postnasal discharge, nasal obstruction, epistaxis or Eustachian tube problems. Diagnosis requires pathologic examination of amyloid deposits. Gross examination may show involved organs to be rubbery with a firm consistency. A waxy grey or yellow appearance is typical. Histologically, amyloid deposits demonstrate eosinophilia after haematoxylin-eosin staining and display apple green birefringence when stained with Congo red and viewed using a polarising microscope [3].
Radiologically, a fluffy appearance in the sinonasal bones adjacent to the amyloid deposit is a specific finding. This may be the result of osteoblastic reaction. Another feature which has been noted is calcification but this is usually considered a non-specific finding [4]. T2 Magnetic resonance imaging (MRI) may show peripheral enhancement in the area around amyloid deposits caused by foreign body giant cell reaction [5]. Lack of enhancement of the amyloid deposits helps to distinguish them from cellular tumours all of which enhance. Key differential diagnoses must also be considered. Idiopathic midline destructive disease (IMDD) has been defined as an unusual progressive condition primarily affecting the nose and paranasal sinuses, sometimes with spread to facial structures, the nasopharynx, orbit, larynx and trachea. However the diagnosis is made only when other causes of midline facial destruction e.g. neoplastic, infective or vasculitic processes have been excluded.

Progressive destruction of the nose and para nasal sinuses may occur with infections like tuberculosis or syphilis and also with fungal pathogens such as aspergillus. Other causes include vasculitic processes like Wegener's disease (granulomatosis with polyangiitis) and neoplasms especially malignant lymphoma. Once infectious causes have been excluded, the main differential diagnoses are Wegener's disease, malignant lymphoma and idiopathic midline destructive disease. Wegener's may show active vasculitis with granuloma in the tissue biopsy. In the presence of pulmonary and renal disease, raised antineurophil cytoplasmic antibody (c ANCA) levels are present in $89 \%$ of cases with active disease [6]. 


\section{Global Journal of Otolaryngology}

\section{Case Report}

A 62-year-old Caucasian male patient presented with complaints of occipital and right temporo-parietal headache along with neck stiffness (not responding to medical treatment with analgesics). The patient also complained of right-sided numbness of the tongue and right otalgia. He gave a history of general malaise and fatigue. There were no complaints of nasal blockage, dysphagia, odynophagia epistaxis or facial pain. His past medical history included clipping of an anterior cranial artery aneurysm in 1995 but he was otherwise fit and well. There was a history of occupational exposure to a sand blasting machine during his work with engine restorations for many years. Thorough ENT examination showed normal ears, oral cavity, oropharynx and larynx. No cervical lympadenopathy was palpable clinically. Both nasal passages were patent and nasal endoscopic examination showed gross crusting of the posterior nasopharyngeal wall (Figure 1).

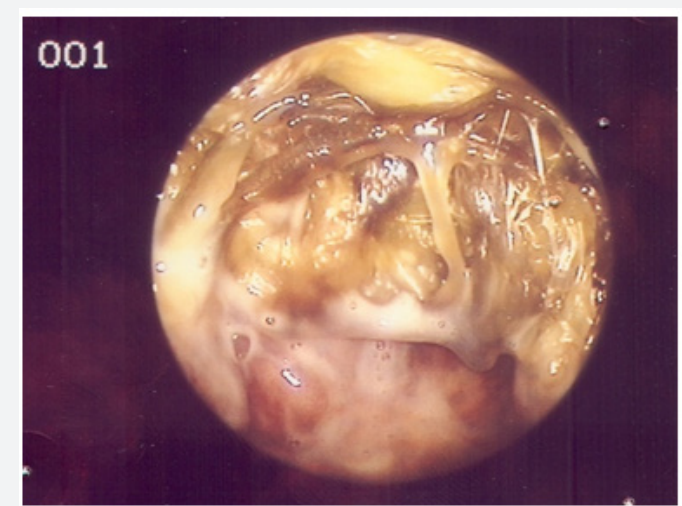

Figure 1: This is a clinical photograph showing gross crusting of the posterior nasopharyngeal wall.

Haematological and biochemical tests showed raised white blood cell count (WBC), erythrocyte sedimentation rate (ESR) and C-reactive protein (CRP) but negative results for all granulomatous markers. Bence Jones protein and serum electrophoresis were negative. A CT (computerised tomography) scan of the head showed a soft tissue mass, crossing the midline in the postnasal space with areas of low attenuation possibly representing necrosis or fluid collection (Figure 2). Intraoperatively, extensive ulceration was noted with pus draining via a sinus in the posterior nasopharyngeal wall (Figure 3 ). This area was swabbed for microbiology and biopsies were sent for histopathology. Microbiology swabs grew staphylococcus aureus, diphtheroids and citrobacter freundii. Further culture grew enterococcus. Histolopathological analysis revealed inflammatory debris and evidence of foreign body reaction with associated bifringent material. These findings were felt to be in keeping with idiopathic midline destructive disease. No evidence of malignancy was reported.

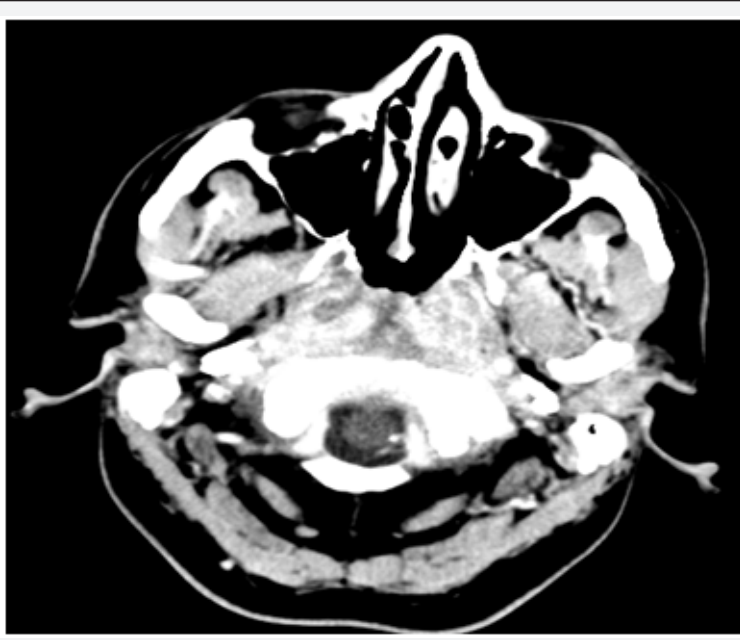

Figure 2: Axial CT (computerised tomography) scan of the head showed a soft tissue mass, crossing the midline in the postnasal space with areas of low attenuation possibly representing necrosis or fluid collection.

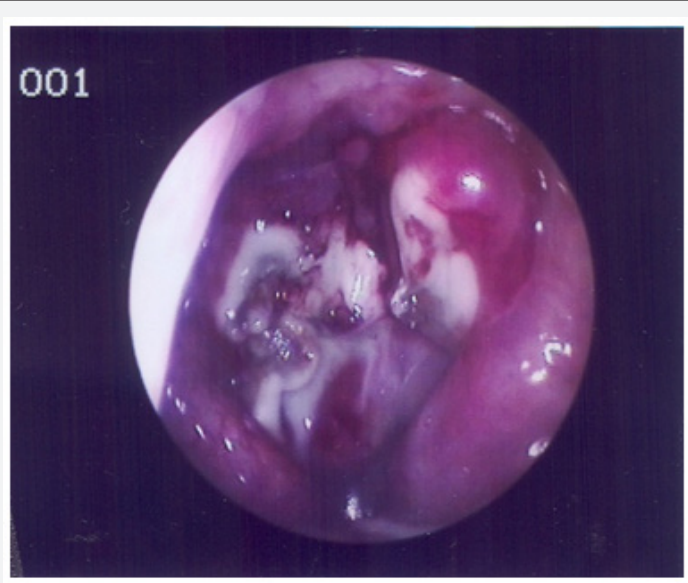

Figure 3: Intra-operative clinical photograph showing extensive ulceration with pus draining via a sinus in the posterior nasopharyngeal wall.

As the patient's symptoms persisted and inflammatory markers increased, he underwent a repeat CT scan (Figure 4). The repeat scan showed bony erosion around the clivus and foramen lacerum. The findings necessitated a second examination under anaesthesia. Again a friable postnasal ulcer with granulations and pus was noted (Figure 5). Repeat biopsies were taken which were reported as granulation tissue and inflammation with amyloid deposition on the basis of special stains (Figures 6-9). Detailed clinical evaluations failed to demonstrate any evidence of systemic amyloidosis. The patient underwent repeated debridement of the ulcer under endoscopic guidance followed by intravenous antibiotics (meropenem) for six weeks and then oral trimethoprim for 4 months. Gradually the inflammatory markers improved and clinically his condition resolved over 6 


\section{Global Journal of Otolaryngology}

months. The last clinical photograph taken at 12 months (Figure 10) showed healthy postnasal space mucosa with no signs of any recurrent disease. The last CT scan also taken at 12 months following initial presentation is shown in Figure 11.
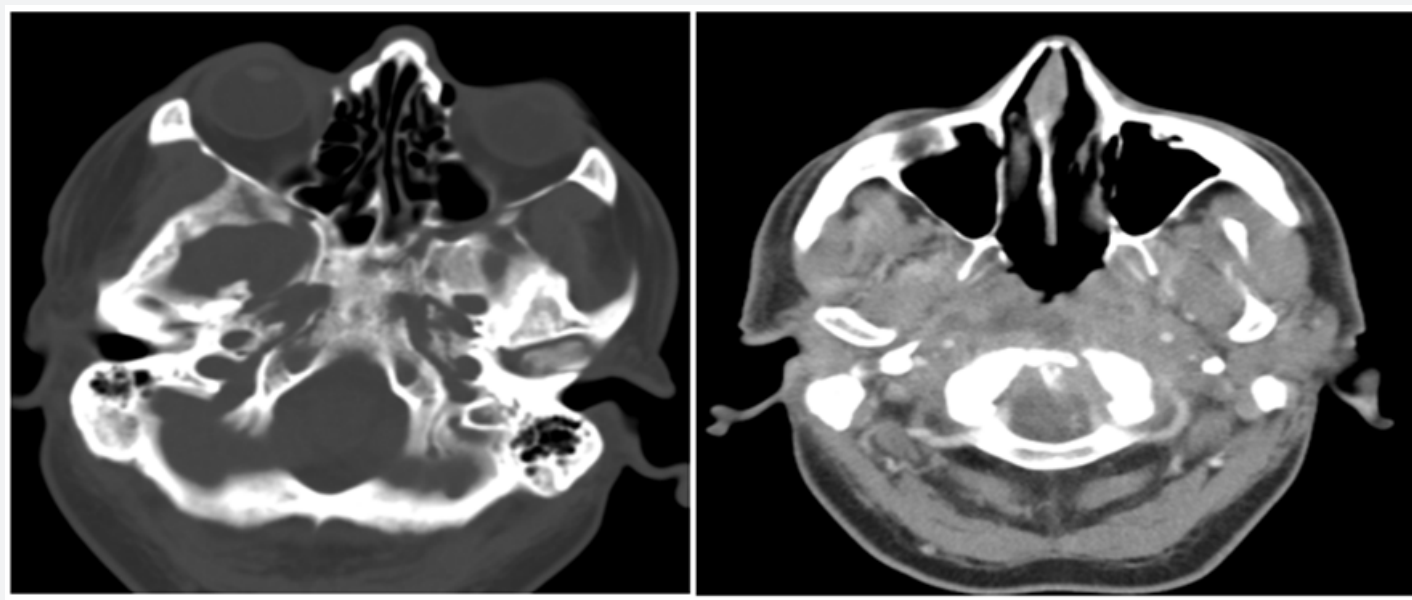

Figure 4: Repeat axial CT scan showing bony erosion around the clivus and foramen lacerum.
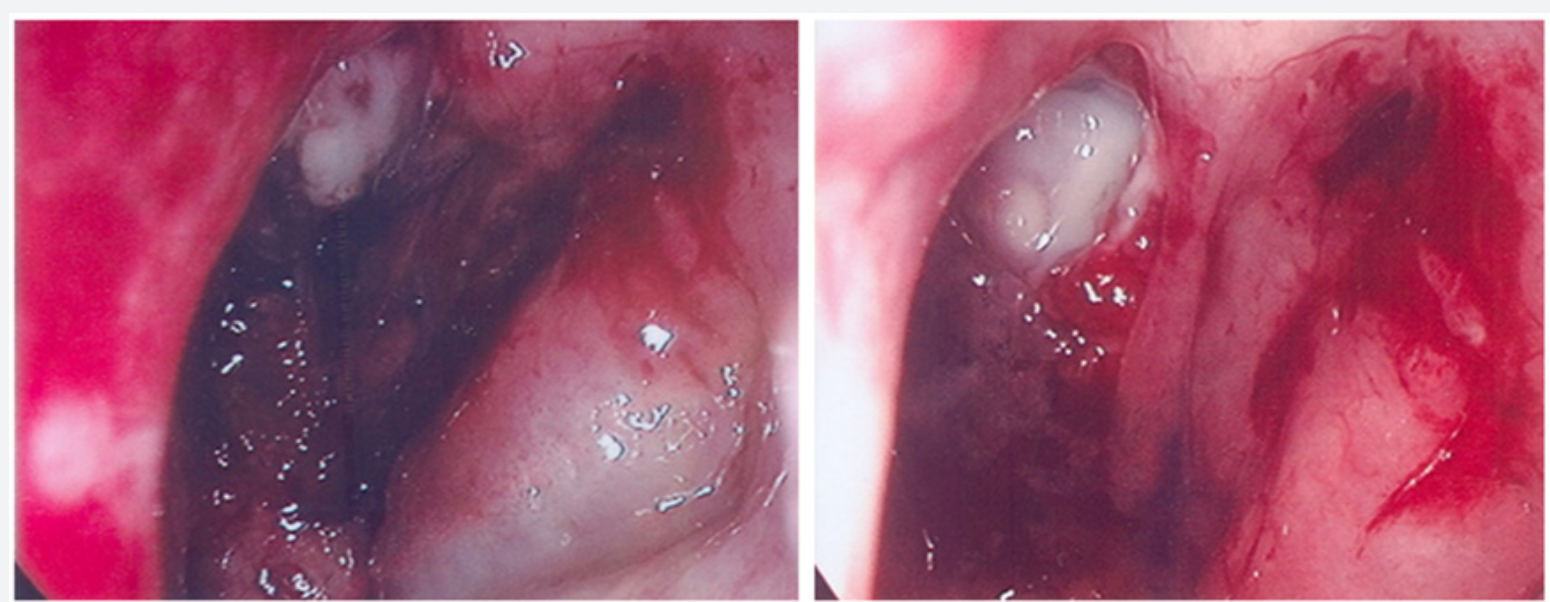

Figure 5: Clinical photograph showing a friable postnasal space ulcer with granulations and pus.

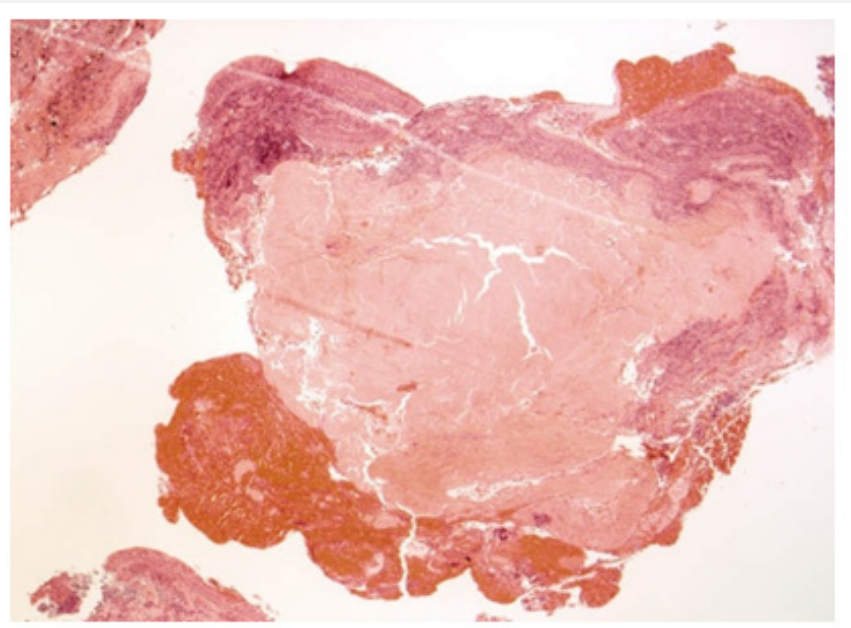

Figure 6: Photomicrograph slide showing posterior nasopharyngeal amyloidosis - H\&E stain x40. 


\section{Global Journal of Otolaryngology}

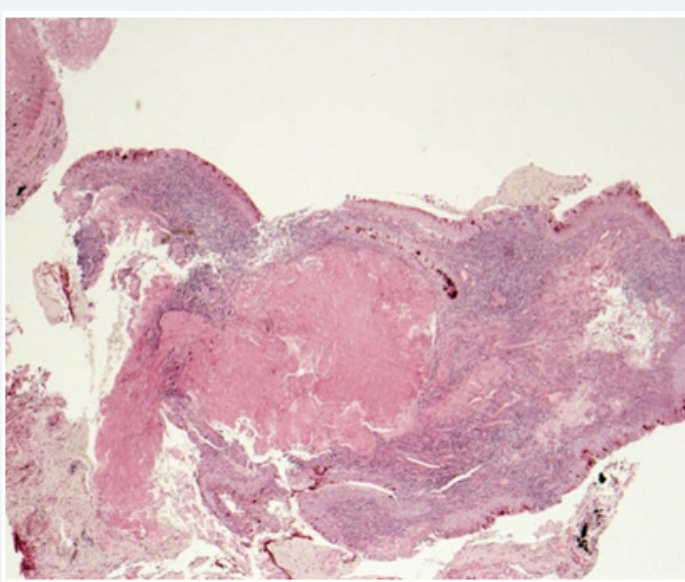

Figure 7: Photomicrograph slide showing posterior nasopharyngeal amyloidosis - PAS stain $\times 40$.

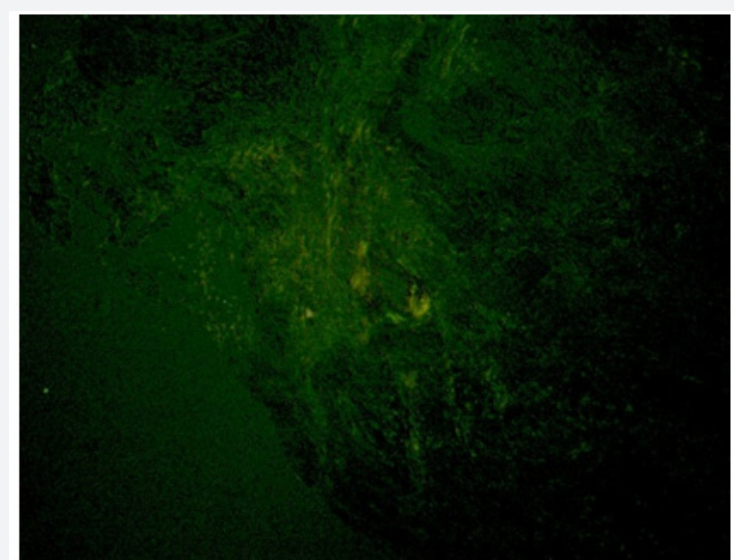

Figure 8: Apple green birefringence of the Congo red- positive amyloid material under polarised light.

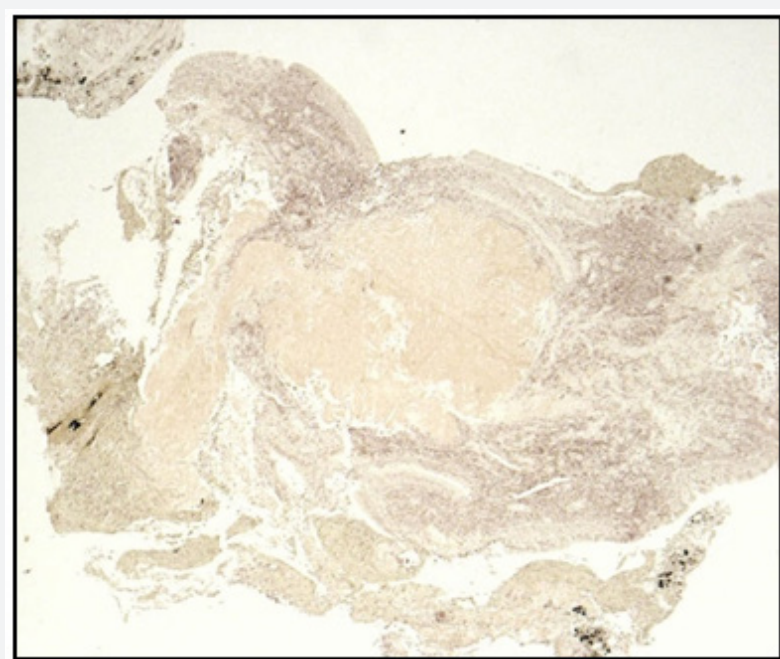

Figure 9: Posterior nasopharyngeal amyloidosis - congo red stain $-x 40$.

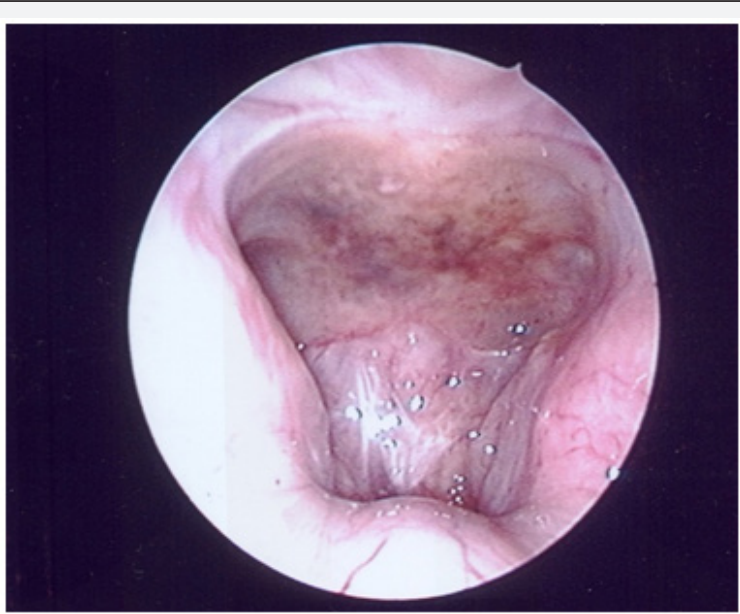

Figure 10: Clinical photograph taken at 12 months showing healthy appearance of postnasal space mucosa with no signs of recurrent disease.

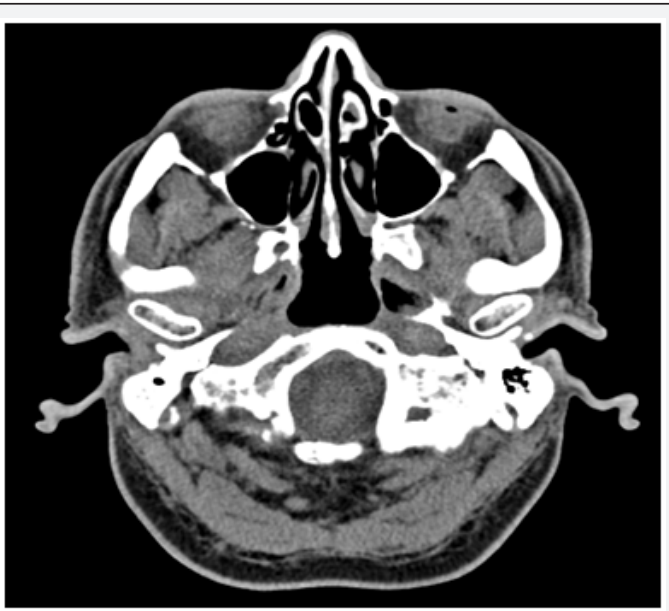

Figure 11: Axial CT scan 12 months following initial presentation showing normal post nasal space appearance.

\section{Discussion}

Amyloid was first described by Rokitansky in 1842. It was not until 1853 that Virshow termed these lesions 'amyloid' in the belief that carbohydrate was the main constituent. Localised amyloidosis is rare. Focal amyloidosis also called amyloidoma are slow growing lesions and are considered benign tumours, but they may be locally aggressive causing osteolysis. To exclude systemic amyloidosis; abdominal, gingival or rectal biopsy are recommended. The clinical significance is that there is shorter life expectancy with the systemic form of amyloidosis [7]. Our reported case, occurring in the nasopharynx, initially appeared to display features of idiopathic midline destructive disease (IMDD). IMDD is diagnosed if the disease is limited to the nose and paranasal sinuses without any evidence of systemic disease at presentation or during follow up. In this condition, characteristic biopsies show non-specific active chronic 
inflammation only with no evidence of amyloid deposition [8]. Other neoplastic, infective or vasculitic processes must have been excluded to make this diagnosis.

In our case report, the finding of amyloid deposits on repeat biopsies was crucial to confirm the diagnosis of amyloidosis.

\section{Conclusion}

Although the nasopharynx has been reported as a site affected by amyloid deposition, we did not come across any case with local amyloid deposits, presenting with skull base erosion in a similar fashion to IMDD. Aggressive debridement along with intravenous antibiotics resolved the condition. It was a difficult condition to diagnose as clinical markers and radiological bony erosion were not in keeping with amyloidosis. Surgery is the most effective treatment for localised amyloidosis if causing morbidity. If the amyloid deposits do not cause any problems then conservative treatment with watchful observation can be considered. As localised amyloidosis is usually an indolent, benign process, surgical intervention must preserve organ function as much as possible. If all serological, morphological, immunohistochemical and molecular biological investigations have been exhausted, the diagnosis of IMDD may be justified.

\section{Acknowledgement}

Mrs Janet Phillips - Medical Photography, The Great Western Hospital, Swindon.

\section{References}

1. Barnes L (2001) Miscellaneous disorders of head and neck. Surgical pathology of head and neck. ( $\left.2^{\text {nd }} E d n\right)$; New York Marcel Dekkar 3: 2191-2193.

2. Raymond AK, Sneige N, Batsakis JG (1992) Amyloidosis in the upper aero digestive tracts. Ann Otol Rhinol Laryngol 101(9): 794-796.

3. Simpson GT, Fuller AP, Strong MS (1984) Localised amyloidosis of the head and neck and upper aero digestive and lower respiratory tracts. Ann Otol Rhinol Laryngol 93(4-1): 374-379.

4. Chin SC, Fatterpeckar G, Kao CH, Chen CY, Som PM (2004) Amyloidosis concurrently involving the sinonasal cavities and larynx. Am J Neuroradiol 25(4): 636-638.

5. Barnes EL Jr, Zafar T (1997) Laryngeal amyloidosis: Clinicalpathologic study of seven cases. Ann Otol Rhinol Laryngol 86: 856-863.

6. Fauci AS (1994) Wegener's granulomatosis: Harrisons Principles of internal medicine. McGraw-Hill Inc, New York, USA, pp. 1674-1676.

7. Panda NK, Sharma SC, Mann SB (1994) Localised amyloidosis of the nasopharynx. Ear Nose Throat J 73(5): 335-336.

8. Tskos M, Fauci AS, Costa J (1982) Idiopathic midline destructive disease IMMD: A subgroup of patients with the midline granuloma syndrome. American Journal of clinical pathology 77(2): 162-168.

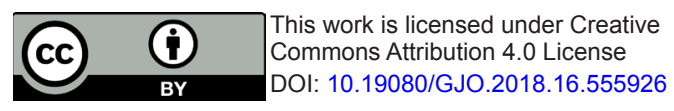

\section{Your next submission with Juniper Publishers will reach you the below assets}

- Quality Editorial service

- Swift Peer Review

- Reprints availability

- E-prints Service

- Manuscript Podcast for convenient understanding

- Global attainment for your research

- Manuscript accessibility in different formats ( Pdf, E-pub, Full Text, Audio)

- Unceasing customer service

Track the below URL for one-step submission https://juniperpublishers.com/online-submission.php 